\title{
Near-field distribution, directivity, and differential scattering cross section of a line source-excited metamaterial-coated electrically small cylinder
}

\author{
Arslanagic, Samel; Ziolkowski, Richard W.; Breinbjerg, Olav
}

Published in:

First European Conference on Antennas and Propagation, 2006. EuCAP 2006.

Link to article, DOI:

10.1109/EUCAP.2006.4585076

Publication date:

2006

Document Version

Publisher's PDF, also known as Version of record

Link back to DTU Orbit

Citation (APA):

Arslanagic, S., Ziolkowski, R. W., \& Breinbjerg, O. (2006). Near-field distribution, directivity, and differential scattering cross section of a line source-excited metamaterial-coated electrically small cylinder. In First European Conference on Antennas and Propagation, 2006. EuCAP 2006. (pp. 1-6). IEEE. https://doi.org/10.1109/EUCAP.2006.4585076

\section{General rights}

Copyright and moral rights for the publications made accessible in the public portal are retained by the authors and/or other copyright owners and it is a condition of accessing publications that users recognise and abide by the legal requirements associated with these rights.

- Users may download and print one copy of any publication from the public portal for the purpose of private study or research.

- You may not further distribute the material or use it for any profit-making activity or commercial gain

- You may freely distribute the URL identifying the publication in the public portal 


\title{
NEAR-FIELD DISTRIBUTION, DIRECTIVITY AND DIFFERENTIAL SCATTERING CROSS SECTION FOR A LINE SOURCE-EXCITED METAMATERIAL-COATED ELECTRICALLY SMALL CYLINDER
}

\author{
Samel Arslanagić ${ }^{(1)}$, Richard W. Ziolkowski( ${ }^{(2)}$, Olav Breinbjerg ${ }^{(1)}$ \\ ${ }^{(I)}$ Ørsted $\bullet D T U$, Electromagnetic Systems, Technical University of Denmark, \\ DK-2800 Kgs. Lyngby, Denmark, Email: sar@oersted.dtu.dk \\ (2) Department of Electrical and Computer Engineering, University of Arizona, \\ Tucson, AZ 85721-0104, USA, Email:Ziolkowski@arizona.ece.edu
}

\begin{abstract}
An antenna configuration consisting of an arbitrarily located electric line source radiating in the presence of concentric metamaterial cylinders is examined. In particular, the near field, the directivity, and the differential scattering cross section are investigated for specific metamaterial structures, and these results are compared to those for the corresponding structures based on double-positive materials. It is shown that specific electrically small metamaterial structures possess radiation and scattering features that surpass those of the corresponding double-positive material structures.
\end{abstract}

\section{INTRODUCTION}

The electromagnetic properties of various classes of metamaterials (MTMs), such as double-negative (DNG) and single-negative (SNG) materials, and combinations of these with conventional double-positive (DPS) materials, have stimulated much recent interest in antenna and device applications. It has been shown that specific MTM-based structures have performance characteristics that significantly surpass those for structures based on DPS materials alone. In particular, the possibility of achieving highly sub-wavelength resonant structures, including waveguides, cavities, scatterers, and radiators, by clever arrangements of DPS, DNG, and/or SNG materials has been demonstrated, see [1] and the works referenced therein as well as [2]-[4]. Among the many interesting features, it has been shown that such structures, despite their electrically small sizes, exhibit significant enhancements of the total radiated power as well as their total scattering cross sections.

In this work, an antenna configuration consisting of an arbitrarily located, infinitely long, electric line source (ELS) radiating in the presence of infinitely long concentric MTM circular cylinders is analysed. It is demonstrated that electrically small versions of these nested structures can be designed to be resonant with the procedures outlined in [3]. The near field, the two-dimensional directivity, and differential scattering cross section, henceforth referred to simply as the directivity and the cross section, respectively, of these resonant designs are examined; and their resonant enhancements, not present in their DPS counterparts, are illustrated. Moreover, it is shown that by positioning the ELS properly with respect to the interfaces between the cylinders, directive electrically small antennas and scatterers can be achieved. This work, in which the dipolar resonance is emphasized, is an extension of [3], where a detailed treatment of the scattering, rather than the antenna, configurations and figures of merit were presented. Throughout this manuscript, the time factor $\exp (j a t)$, with $\omega$ being the angular frequency, and $t$ being the time, is assumed and suppressed.

\section{CONFIGURATION AND THEORY}

While the details of the analytical solution, to which frequent reference is made in the following, can be found in [3], the main points are summarized below. Figure 1.

The configuration of interest is depicted in

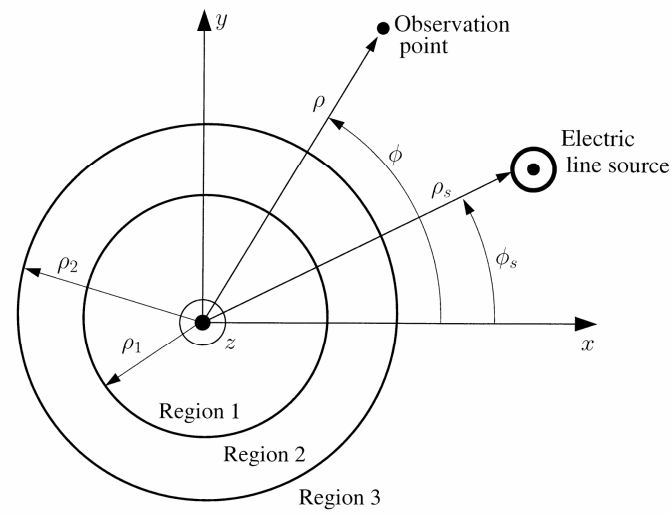

Figure 1. The configuration: concentric MTM cylinders illuminated by an ELS.

It consists of a pair of infinite, concentric, circular cylinders; i.e., a central core (region 1 ) of radius $\rho_{1}$, covered with a layer (region 2 ) of radius $\rho_{2}$, surrounded by free space (region 3). Regions 1 and 2 are composed of simple and lossless DPS, DNG, and/or SNG 
materials. Related results for dispersive MTMs have been presented in [3]. The structure is illuminated by an infinite ELS of constant electric current $I_{e}$ [A]. The axes of the cylinders and the ELS, which can be located in any of the three regions, are parallel. Region $i$, with $i=1,2,3$, is characterized by a permittivity, $\varepsilon_{i}$, and a permeability, $\mu_{i}$, and the wave number $k_{i}=\omega \sqrt{\varepsilon_{i} \mu_{i}}$, where the branch of the square root is chosen such that $k_{i}>0$ in a DPS material $\left(\varepsilon_{i}>0, \mu_{i}>0\right), k_{i}<0$ in a DNG material $\left(\varepsilon_{i}<0, \mu_{i}<0\right)$, and $\operatorname{Im}\left\{k_{i}\right\} \leq 0$ in a SNG material (either $\varepsilon_{i}$ or $\mu_{i}$ is negative). A cylindrical coordinate system $(\rho, \varphi, z)$ and the associated Cartesian coordinate system $(x, y, z)$ are introduced such that the $z$-axis coincides with the common axis of the cylinders. The coordinates of the observation point and the ELS are $(\rho, \varphi)$ and $\left(\rho_{s}, \varphi_{s}\right)$, respectively.

The field generated by the ELS in an infinite medium characterized by $\varepsilon_{i}, \mu_{i}$, and $k_{i}$ is given by the following well-known expansion in terms of cylindrical wave functions [5]

$$
\begin{aligned}
& \vec{E}_{E L S}(\rho, \varphi)=-\hat{z} I_{e} \frac{\omega \mu_{i}}{4} \times \\
& \left\{\begin{array}{l}
\sum_{n=0}^{N_{\max }} \varepsilon_{n} J_{n}\left(k_{i} \rho\right) H_{n}^{(2)}\left(k_{i} \rho_{s}\right) \cos \left[n\left(\varphi-\varphi_{s}\right)\right], \rho \leq \rho_{s} \\
\sum_{n=0}^{N_{\max }} \varepsilon_{n} J_{n}\left(k_{i} \rho_{s}\right) H_{n}^{(2)}\left(k_{i} \rho\right) \cos \left[n\left(\varphi-\varphi_{s}\right)\right], \rho \geq \rho_{s}
\end{array},\right.
\end{aligned}
$$

where $J_{n}(\cdot)$ is the Bessel function of order $n$ and is chosen to represent the field for $\rho \leq \rho_{s}$ due to its nonsingular behavior at the origin, while $H_{n}^{(2)}(\cdot)$ is the Hankel function of second kind and order $n$, and is chosen for $\rho \geq \rho_{s}$ because it represents an outward propagating wave that complies with the radiation condition. The symbol $\varepsilon_{n}$ is the Neumann number, i.e., $\varepsilon_{n}=1$ for $n=0$, and $\varepsilon_{n}=2$ otherwise. Furthermore, $N_{\max }$ is the truncation limit and is chosen to ensure convergence of the cylindrical wave expansions.

The scattered field in the region containing the ELS and the total fields in the other regions are also expanded in terms of cylindrical wave functions. These fields involve unknown expansion coefficients that are found as a solution of the relation [2]

$$
\vec{C}_{n}=\left(\overline{\bar{M}}_{n}\right)^{-1} \vec{\Lambda}_{n}, \quad n=0,1, \ldots, N_{\max }
$$

where $\vec{C}_{n}=\left[C_{1 n}, C_{2 n}, C_{3 n}, C_{4 n}\right]$ is the vector containing the four unknown coefficients associated with the fields in the three regions, and $\vec{\Lambda}_{n}=\left[\Lambda_{1 n}, \Lambda_{2 n}, \Lambda_{3 n}, \Lambda_{4 n}\right]$ is the excitation vector that depends on the location of the ELS. The matrix $\overline{\bar{M}}_{n}$ is a 4-by-4 matrix that arises from the enforcement of the electromagnetic boundary conditions and, hence, depends on the values of the cylindrical waves at the two interfaces. For brevity, the explicit forms of this matrix and the excitation vector are not included here.

In [3], specific attention was devoted to a quantity referred to as the power ratio (PR), which is the ratio of the total power radiated by the ELS in the presence of MTM cylinders to the power radiated by the ELS in free space. In terms of the field expansion coefficients, the $\mathrm{PR}$ reads

$$
\mathrm{PR}=\frac{1}{2} \sum_{n=0}^{N_{\max }} \varepsilon_{n}^{2}\left(3-\varepsilon_{n}\right)\left|\alpha_{n}\right|^{2}
$$

Based on Eq. (3), large enhancements of the radiated power were found in [3] for specifically designed MTM-based structures. In the present manuscript, the analysis of these structures is continued for their directivity, $D$, which can be expressed as

$$
D(\varphi)=\frac{2\left|\sum_{n=0}^{N_{\max }} \varepsilon_{n} j^{n} \alpha_{n} \cos \left[n\left(\varphi-\varphi_{s}\right)\right]\right|^{2}}{\sum_{n=0}^{N_{\max }} \varepsilon_{n}^{2}\left(3-\varepsilon_{n}\right)\left|\alpha_{n}\right|^{2}} .
$$

Furthermore, when the ELS is in region 3, the cross section, $\sigma_{d}$, defined by the square of the ratio of the magnitude of the scattered far field to the magnitude of the free-space incident field at the origin, given by

$\sigma_{d}(\varphi)=\frac{4}{k_{3}} \frac{\left|\sum_{n=0}^{N_{\max }} C_{4 n} \varepsilon_{n} j^{n} \cos \left[n\left(\varphi-\varphi_{s}\right)\right]\right|^{2}}{\left|H_{0}^{(2)}\left(k_{3} \rho_{s}\right)\right|^{2}}$.

is investigated. In Eqs. (3) and (4), the quantity $\alpha_{n}=J_{n}\left(k_{3} \rho_{s}\right)+C_{4 n}$ if the ELS is in region 3, while $\alpha_{n}=C_{4 n}$ if the ELS is in any other region, with $C_{4 n}$ being the expansion coefficient for region 3 .

\section{RESULTS AND DISCUSSION}

Next, numerical results for specific structures are presented and analysed. Only DPS and DNG materials are emphasized. Throughout the discussion, a structure is referred to by its properties in regions 1 and 2. For instance, a DPS-DNG structure indicates that region 1 (2) consists of a DPS (DNG) material. 


\subsection{Introductory remarks}

The PR was thoroughly examined in [3] for specific resonant MTM-based structures, for which significant enhancements of the radiated power were found. In particular, the so-called dipolar and quadrupolar structures, i.e., those for which the dominant modes of radiation are $n=1$ and $n=2$, respectively, were studied. For a frequency of operation $f_{0}=300 \mathrm{MHz}$, and the material parameters in various regions selected as $\varepsilon_{1}=\varepsilon_{3}=\varepsilon_{0}, \varepsilon_{2}= \pm \varepsilon_{0} \quad$ and $\quad \mu_{1}=\mu_{3}=\mu_{0}$, $\mu_{2}= \pm 4 \mu_{0}$, with $\varepsilon_{0}$ and $\mu_{0}$ denoting the free-space permittivity and permeability, the geometrical parameters for the resonant dipolar structure where found to be $\rho_{1}=6 \mathrm{~mm}$ and $\rho_{2}=10.03 \mathrm{~mm}^{1}$. The negative (positive) parameters in region 2 apply for a DNG (DPS) material. Thus, the specific antenna configurations under numerical investigation are a DNG or a DPS shell in the presence of arbitrarily located ELS.

In Figs. 1 to 3 , the PR is depicted as a function of the ELS location, $\rho_{s}$, in regions 1, 2, and 3, respectively (in the forthcoming results, the ELS is displaced along the $x$-axis to a distance $\rho_{s}$, i.e., its coordinates are $\left.\left(\rho_{s}, \varphi_{s}=0^{\circ}\right)\right)$. For the DPS-DNG structure, the largest PR occurs in all cases when the ELS is located close to either of the two surfaces of the DNG shell. For the ELS in region 1, the enhancements are diminished as the ELS is moved toward the origin and, as noted in [3], are completely lost when the ELS is at the origin or very close this. This is due to the fact that the $n=0$ (monopolar) mode increasingly dominates for smaller and smaller $\rho_{s}$ values, and it is the only radiating mode when $\rho_{s}=0$. For the ELS in region 3, the PR is decreasing for increasing $\rho_{s}$ values. For a majority of the ELS locations in region 2, large PRs are obtained. However, for $\rho_{s}=7.746076 \mathrm{~mm}$ no enhancement occurs. As will be demonstrated below via the near-field investigations, the monopolar mode is dominant in this case, and as shown in [2], no resonance can occur for the monopolar mode. It thus follows that in order to obtain the enhancements, the higher order modes need to be excited. In none of the electrically small cases does the corresponding DPS-DPS structure lead to any enhancements of the radiated power.

\subsection{Near field}

In Figs. 5 through 9, the quantity $20 \log _{10}|\vec{E}(\rho, \varphi)|$, where $\vec{E}$ denotes the total electric field normalized by

\footnotetext{
${ }^{1}$ Here, only the resonant dipolar mode is considered, while the influence of e.g., the quadrupolar mode will be reported elsewhere.
}

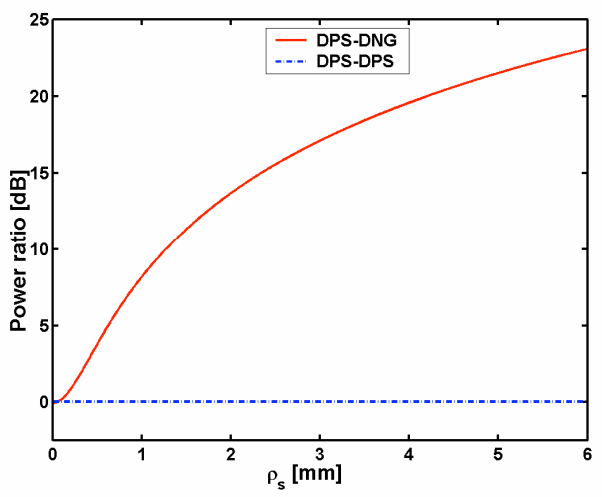

Figure 2. PR as function of $\rho_{s}$. ELS is in region 1 .

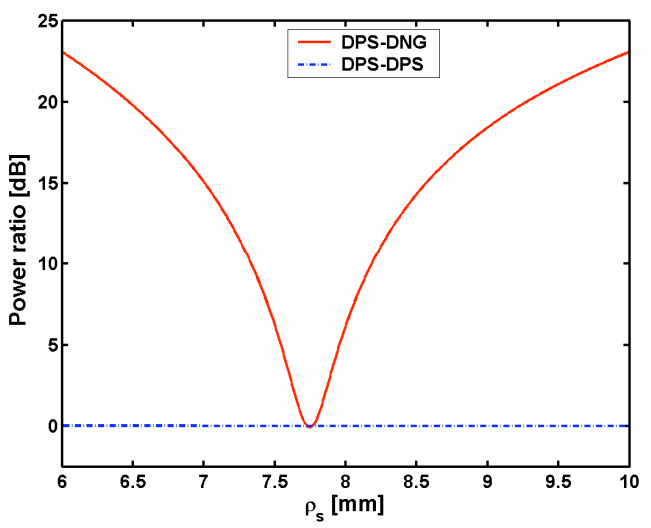

Figure 3. PR as function of $\rho_{s}$. ELS is in region 2.

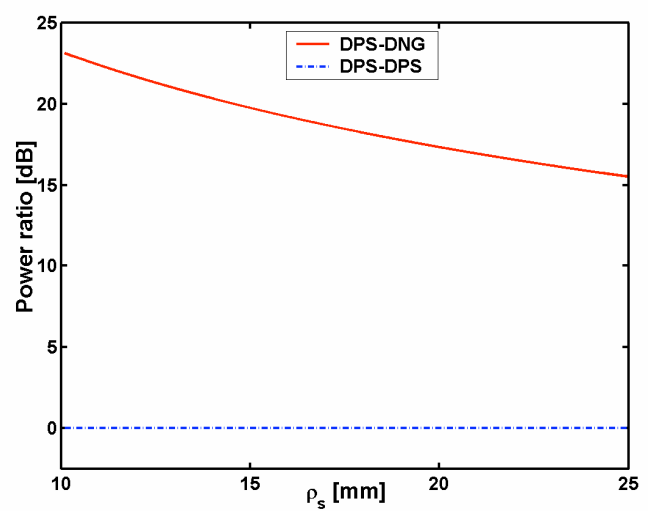

Figure 4. PR as function of $\rho_{s}$. ELS is in region 3.

$1 \mathrm{~V} / \mathrm{m}$, is shown in a circular region of radius $3 \rho_{2}$ (only the results for the DPS-DNG structure are shown since the corresponding DPS-DPS structure always yields a monopolar pattern). While Figs. 5-7 illustrate the field behaviour when the ELS is in region 1, Fig. 8 does so when the ELS is in region 2. When the ELS is at $\rho_{s}=0.001 \mathrm{~mm}$, the near field has the monopolar form, see Fig. 5. This confirms that for locations of the ELS close to the origin, the monopolar mode is dominant. As a consequence, no enhancement of the radiated 
power is expected for such ELS locations. In [3], the analytical solution revealed that only the monopolar mode radiates for $\rho_{s}=0$. As the ELS is moved just slightly away from the origin, the monopolar mode is mixed with the dipolar mode, as confirmed by Fig. 6, which shows the near field for $\rho_{s}=0.125 \mathrm{~mm}$. Moving the ELS even further from the origin, e.g., to $\rho_{s}=1 \mathrm{~mm}$, leads to the near field shown in Fig. 7 that has a form, which is clearly dominated by the dipolar mode. At this ELS location an enhancement of some $8 \mathrm{~dB}$ of the radiated power is in evidence in Fig. 2. These dipolar mode enhancements become even more pronounced as the ELS is moved closer to the DNG shell, e.g., to $\rho_{s}=5.99 \mathrm{~mm}$, where the dipolar mode is excited most strongly. This stronger excitation of the dipolar mode as the ELS is moved closer to the DNG shell is also responsible for the reduction of the asymmetry in the total field, which is present in Fig. 7 and is caused by the off-set nature of the ELS.

If the ELS is in one of the other regions, the near fields have a dipolar form when the ELS is located near the surfaces of the DNG shell. This indicates that the maximum radiated power shown in Figs. 2-4 is a

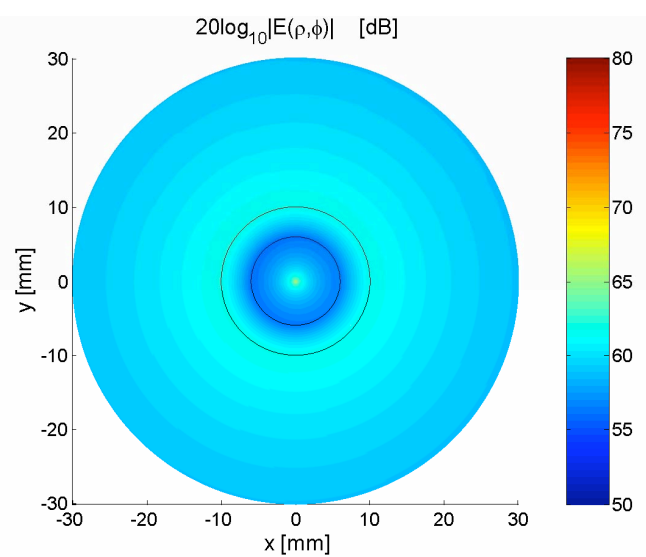

Figure 5. Near field for $\rho_{s}=0.001 \mathrm{~mm}$.

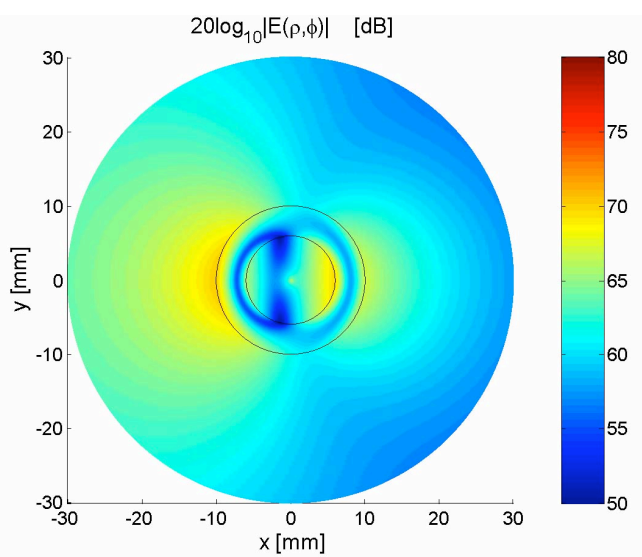

Figure 6. Near field for $\rho_{s}=0.125 \mathrm{~mm}$.

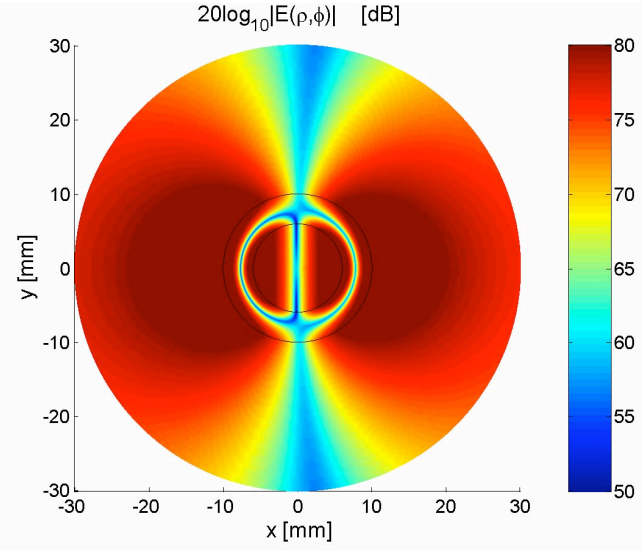

Figure 7. Near field for $\rho_{s}=1 \mathrm{~mm}$.

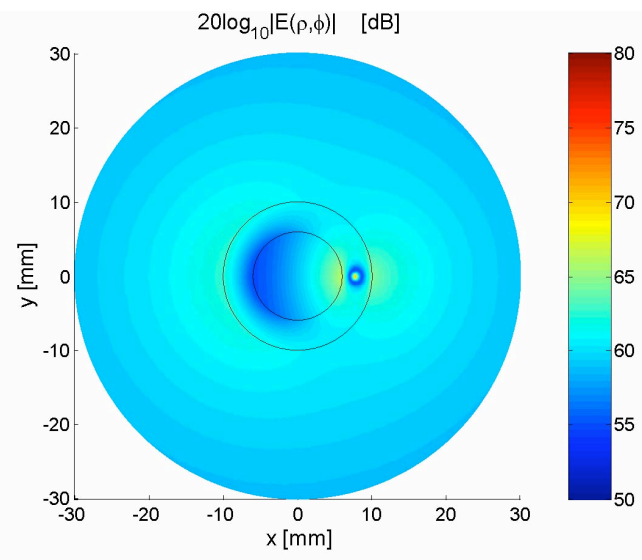

Figure 8. Near field for $\rho_{s}=7.746076 \mathrm{~mm}$

result of the ELS exciting a resonant dipolar mode When the ELS is in region 3 and is moved further away from the DNG shell, the excitation of the dipolar mode is gradually suppressed for the range of $\rho_{s}$ values investigated here, while that of the monopolar mode is increased. This explains the behaviour shown in Fig. 4.

As revealed by Fig. 3, the PR of the resonant DPS-DNG structure is altered significantly as the ELS is moved through region 2. In particular, no enhancement is found for $\rho_{s}=7.746076 \mathrm{~mm}$. The near field for this particular location is depicted in Fig. 8 where a clear monopolar form is observed for the observation points located farthest from the origin. Thus, the ability of the ELS to excite the dipolar mode in this resonant DPS-DNG structure changes significantly as it is moved though the DNG shell, and is, in fact lost, for that particular ELS location. The observation of the monopolar mode for $\rho_{s}=7.746076 \mathrm{~mm}$ is rather interesting since the present DPS-DNG structure was designed specifically to excite the dipolar mode as the dominant one. Its observation suggests that close to this ELS location the near field is a mixture of a dipolar and stronger monopolar mode, which results in the reduced 
enhancement of the radiated power observed in Fig. 3. Similar gradual transformations of the near field have been observed in Figs. 5 and 6 for the ELS locations close to the origin. These gradual transformations of the pattern as the ELS moves in different regions will be analysed further in Sec. 3.2.

\subsection{Directivity}

Figure 9 shows the directivity for a number of ELS locations for the DPS-DNG and DPS-DPS structures. For the DPS-DNG structure, the results are in very good agreement with the near-field results discussed above. In particular, close to the origin, i.e., for $\rho_{s}=0.001 \mathrm{~mm}$, the directivity is dominantly monopolar. However, for $\rho_{s}=0.125 \mathrm{~mm}$, the directivity has a form, which is a mixture of the monopolar and dipolar modes, with the former one being the dominant one. For $\rho_{s}=1$ and $5.99 \mathrm{~mm}$, the directivity takes on the dipolar form, although not entirely symmetric. It is easily observed that the asymmetry in the pattern is reduced as the ELS is moved toward the DNG shell, this likewise being in agreement with the near field results. In comparison, the directivity of the corresponding DPS-DPS structure has a monopolar form, regardless of the ELS location. Thus, apart from enhanced PR values, the DPS-DNG structure provides one with the possibility of reshaping the pattern from the monopolar form to the dipolar one simply by changing the location of the ELS.

When the ELS is in region 2, the distinctive directivities of the dipolar form are found for the DPS-DNG structure when the ELS is located near either of the interfaces of the DNG shell, while the directivity is perfectly monopolar for $\rho_{s}=7.746076 \mathrm{~mm}$. This behaviour is illustrated in Fig. 10 and is in agreement with the PR and near-field results. It is linked to the previously mentioned gradual transition from the dipolar to the monopolar and back to the dipolar mode of radiation, and is further illustrated in Fig. 11, where the directivity is given for various ELS locations slightly to the left and right of $\rho_{s}=7.746076 \mathrm{~mm}$. For $\rho_{s}=7.5 \mathrm{~mm}$, the directivity takes on the dipolar form for which the main lobe is in the $\varphi=180^{\circ}$ direction, while for $\rho_{s}=7.7 \mathrm{~mm}$, it is close to the monopolar form. This is also the case for $\rho_{s}=7.8 \mathrm{~mm}$, while for $\rho_{s}=8 \mathrm{~mm}$, the directivity again has a dipolar form but now with the main lobe in the $\varphi=0^{\circ}$ direction. Thus, the main lobe of the recorded directivities of the dipolar form is in the same direction as the one in which the ELS is displaced from $\rho_{s}=7.746076 \mathrm{~mm}$. In regards to these observations of asymmetry, one also notes that with the ELS being displaced along the $x$-axis, the patterns found on both sides of $\rho_{s}=7.746076 \mathrm{~mm}$ are

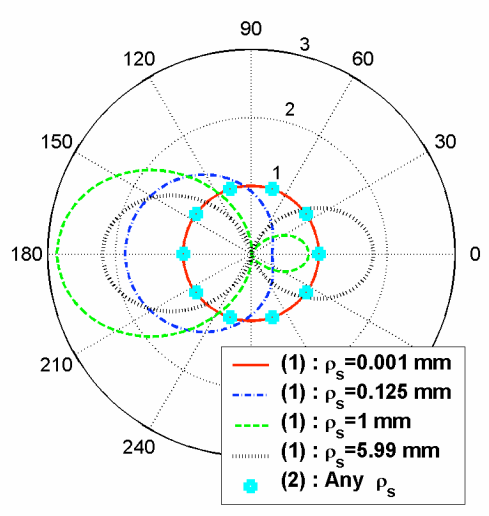

Figure 9. Directivity for DPS-DNG (1) and DPS-DPS (2) structures for various ELS locations in region 1.

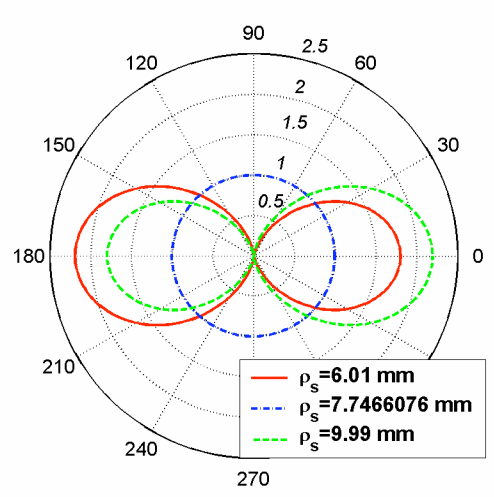

Figure 10. Directivity for the DPS-DNG structure for various ELS locations in region 2.

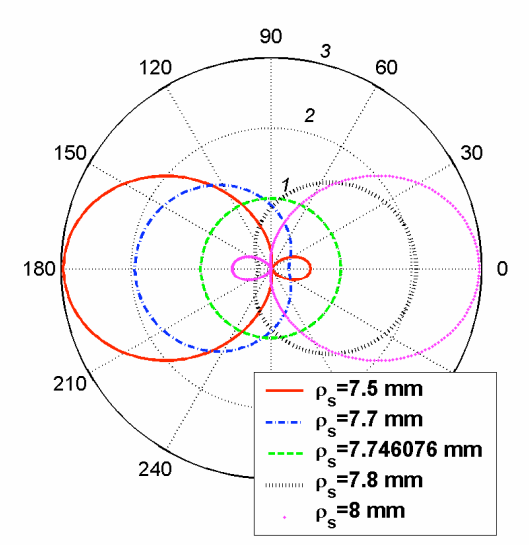

Figure 11. Directivity for the DPS-DNG structure for various ELS locations around $\rho_{s}=7.746076 \mathrm{~mm}$.

mirror images of each other with respect to the $y$-axis. The results of Figs. 10 and 11 clearly illustrate how the directivity is reshaped from the dipolar to monopolar, and back to the dipolar form, this being in agreement 
with Fig. 3. When the ELS is in region 3 at $\rho_{s}=10.1$, 15,20 and $30 \mathrm{~mm}$ respectively, the directivity takes on the dipolar forms shown in Fig. 13. Within the range of $\rho_{s}$ values investigated here, more power is radiated in the $\varphi=0^{\circ}$ direction and the pattern becomes increasingly asymmetric as the ELS moves further away from the DNG shell.

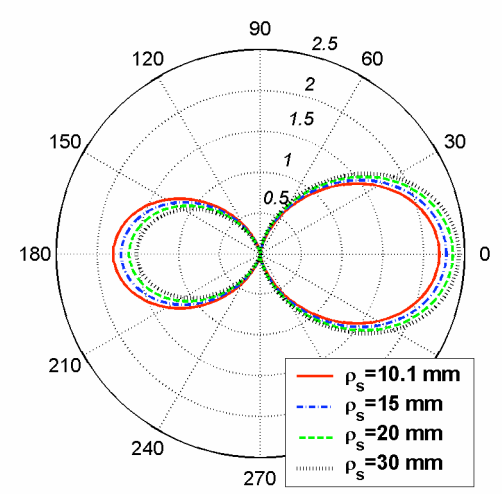

Figure 12. Directivity for the DPS-DNG structure for various ELS locations in region 3.

\subsection{Cross section}

The cross section, given by Eq. (5), is depicted in Figs. 13 and 14 for the DPS-DNG and DPS-DPS structures, respectively, for various locations of the ELS in region 3. First, all of the patterns shown in Figs. 13 and 14 have a dipolar form since the cross section only uses the scattered field terms. Second, for all of the ELS locations considered, the cross section of the resonant DPS-DNG structure is found to be considerably larger than that of the corresponding DPS-DPS structure. This implies that the DPS-DNG structure at hand, although electrically very small, does exhibit characteristics generally associated to large scatterers. This is in agreement with the PR and the total scattering cross section results reported in [3].

\section{SUMMARY AND CONCLUSIONS}

An antenna configuration consisting of an arbitrarily located ELS radiating in the presence of concentric MTM cylinders was examined. Significant enhancements of the radiated power, as compared to the power radiated in free space, were found for DNGbased structures, while no enhancements were found for the corresponding DPS-based structures. These enhancements were shown to be related to the excitation of the resonant dipolar mode; a fact which was carefully verified with near-field investigations. Moreover, the directivity and the cross section were likewise investigated, and they were also found to exhibit enhancements for the DNG-based structures, while none were observed for the corresponding DPS-based structures. Furthermore, the possibility of pattern reshaping by a proper positioning of the ELS was demonstrated, thus suggesting the feasibility of controlling the directivity of electrically small MTMbased antennas.

The effects of losses as well as the use of SNG materials will be reported elsewhere in the near future.

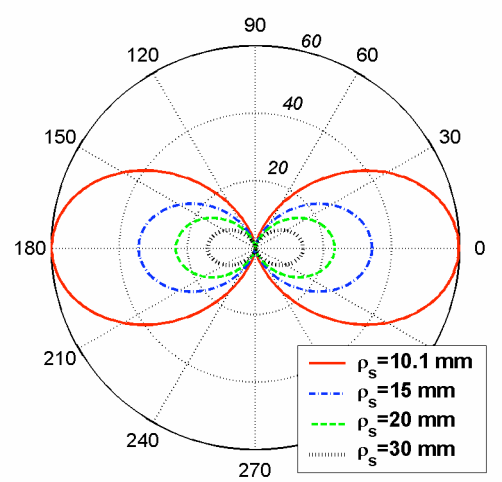

Figure 13. Cross section for the DPS-DNG structure for various ELS locations.

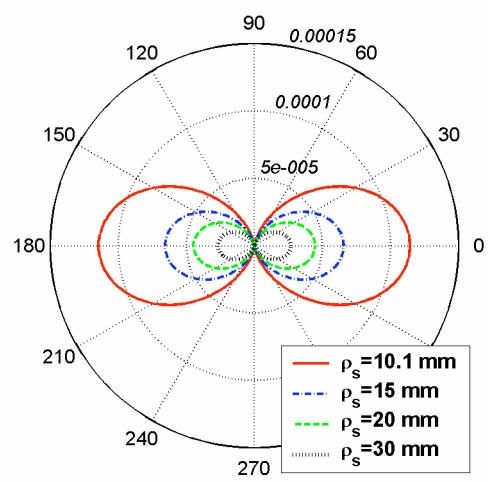

Figure 14. Cross section for the DPS-DPS structure for various ELS locations.

\section{REFERENCES}

1. N. Engheta and R. W. Ziolkowski, IEEE Microwave Theory Tech., 53, pp. 1535-1556 (2005).

2.S. Arslanagić, R.W. Ziolkowski, Olav Breinbjerg, IEEE AP-S International Symposium, Albuquerque, NM, USA, 2006 - accepted.

3. -, Mircowave Opt. Technol. Lett., 2006 - accepted.

4.-, IV. Int. Workshop on EM Wave Scattering, Turkey, 2006 - accepted.

5.C. A. Balanis, Advanced Engineering Electromagnetics, John Wiley \& Sons, 1989. 